\title{
Researching Migration in a Superdiverse Society: Challenges, Methods, Concerns and Promises
}

\author{
by Lisa Goodson and Aleksandra Grzymala-Kazlowska \\ University of Birmingham; University of Birmingham
}

Sociological Research Online, 22 (1), 2

<http://www.socresonline.org.uk/22/1/2.html>

DOI: $10.5153 /$ sro.4168

Received: 27 Aug 2015 | Accepted: 21 Nov 2016 I Published: 28 Feb 2017

\begin{abstract}
The arrival of superdiversity raises a wide range of methodological issues that warrant further consideration by social researchers conducting research in superdiverse contexts. The complex multi-layering of population settlement that has emerged due to successive waves of migration means that identities, lived experience and access to services including welfare are played out in a plethora of different ways, often determined by the interplay of a range of socio-economic variables alongside structural characteristics, which influence the fundamental rights and entitlements of individuals living in the UK and in turn their settlement and adaptation experiences. This paper reflects on the limitations of ethno-centric research designs, which concentrate on ethnicity as the single most important unit of analysis, and calls for more participatory and multidimensional methodologies that engage diverse participants and reflect the levels of socio-demographic complexity experienced in urban areas of society. It then moves on to discuss a number of specific methodological challenges associated with complex populations. In particular sampling and access issues associated with diverse migrant populations will be considered. The latter part of this paper discusses the adoption of a range of research approaches that offer promising potential in terms of better capturing and understanding the heterogeneity, complexity and fluidity concomitant with superdiversity as well as engaging a range of community stakeholders in the production of knowledge.
\end{abstract}

\section{Keywords: Superdiversity, Co-Production Community Research, Maximum Variation Sampling, Respondent Driven Sampling, Community Research, Documents of Life}

\section{Introduction}

Whilst it is acknowledged that in the past 20 years the United Kingdom, along with many other European countries, has entered an era described as 'superdiversity' (Vertovec 2007, 2010), social researchers have only recently begun to reflect on the implications of this rapid societal change in relation to the scope and design of their research. The changes brought about by superdiversity demand the development of new methodologies and ways of thinking about research that can inform new theory creation, policy and practice.

1.2 The notion of superdiversity has been used to describe socio-cultural and demographic complexity driven by international migration and internal differentiation within societies. This socio-cultural and demographic complexity has taken place on a larger scale, at a greater speed and spread across wider geographical areas than in the past. In short, superdiversity refers to levels of diversity which supersede anything previously experienced (Vertovec 2012). In the UK, for example, the increase in new migrants during the 1990s, including both asylum seekers and refugees, and economic migrants from A8 and A2 countries ${ }^{[1]}$ following the expansion of the European Union, has contributed to the population complexity which Vertovec (2010) and others (Phillimore 2015; Blommaert 2013a and b; Sepulveda et al. 2011; Wessendorf 2010) associate with superdiversity. Analysis of the 2011 census data shows that the preceding decade witnessed the largest number of newcomer arrivals to the UK ever. Over with $13 \%$ of the population (one in eight people) are now born overseas, which equates to $7,505,010$ individuals (ONS 2013). 
such as London or Birmingham is a population that is 'multi-layered' with 'indigenous', 'old migrant' and 'new migrant' populations living side by side (Phillimore 2015). As a result the socio-demographic makeup of urban communities has become more complex as we see the settlement of a broader range of people than ever before. Individuals with different ethnicities, age groups, genders, classes, faith denominations, immigration statuses and reasons for migrating now make up the new migrant population. Superdiversity is mainly associated with urban settings, particularly global cities, since as Sassen (1996: 188) notes, cities 'concentrate diversity'. The new derivative term 'superdivercity' has even been coined and used for example at recent events such as the 'Academy of Urban Super-Diversity' held in Berlin in 2015.

Migration patterns have also become more diverse, with contemporary migration flows encompassing short term and circular or repeat migrants; long-term and settlement migrants, onward migrants and return migrants as well as re-emigrants. These different types of migration patterns all contribute to the emergence of 'supermobile' populations (Urry 2000). Supermobility leads to high levels of tenancy turnover and movement at a neighbourhood level, which has significant implications for research, especially for longitudinal studies, as high levels of attrition are likely to occur when participants relocate out of the area. Furthermore, Engbersen at al. (2013) distinguish different types of contemporary migration on the basis of a relationship between attachment to the country of origin and attachment to a destination country. The different types of migration include: fluid migrants (neither attached to the country of origin nor to a destination state), transmigrants (attached to both of them), short-term or circular migrants (strongly attached to a home country, not to the destination country) and settlement migrants (strongly attached to a receiving state, maintaining limited, if any, ties with the country of origin). This typology highlights not only the possible multiple affiliation of contemporary migrants but also growing numbers of people living on the move. Superdiversity, together with supermobility and transnationalism, presents particular challenges for researchers. Not only is spatially localised data difficult to obtain, due to mobility issues as migrants increasingly operate in complex and changing settings, but research findings quickly become out of date.

1.5 It should be pointed out that superdiversity represents more than a descriptive term capturing a changing demographic reality and pinpointing unprecedented diversity which has become a fact in many places around the world. The emergence of superdiversity debates can be understood in the context of UK/EU equality legislation and heightened sensitivity to issues of equality, not just increased diversity brought by new migration flows (Vertovec 2011). Superdiversity can also be seen as a sensitising concept or more broadly as a theoretical framework that stimulates new ways of thinking and understanding of society. Furthermore, superdiversity may be perceived as a methodological and epistemological approach linked closely to the constructivist paradigm. Moreover, it may be linked to a political programme celebrating and promoting diversity as well as a new 'narrative' which could replace the contested notion of multiculturalism (Vertovec 2007).

In spite of its stimulating value and possible multiple functions and premises, superdiversity is not a widely accepted concept and evokes criticism. Critiques have focused attention on the novelty of superdiversity (Blommaert 2013b); its descriptiveness resulting from ontology-driven research underlining growing complexity and uncertainty in diversity classifications (Arnaut and Spotti 2014). Others have been concerned with the overemphasis on cultural and localised differences at the expense of structural inequalities and politicised retreat from multiculturalism (Sepulveda et al.2011). Back (2015) points out superdiversity fails to address racial issues, social conflicts and divisions; moreover the 'emphasis on superlative difference feeds the fire of public anxieties of an already panicked debate about immigration.' Berkeley (cited in Humphris 2015) argues that the way the term superdiversity has been translated into academic or policy debates may be potentially detrimental to the fight for racial equality because of its focus on fragmentation and promoting diversity rather than social justice. According to Demir (cited in Humphris 2015) this can be linked to the fact that the diversity approach lacks two key elements relating to theories of race: 'recognition', as race helps people make sense of their social and historical position and 'solidarity', as race carries a power to mobilise groups. It may be helpful to reconceptualise race and ethnicity in ways that resonates with people's lives and focuses on the production of race through human agency and social contexts (Knowles 2010) and "everyday life" (Smith 2014). Another weakness of the concept of superdiversity is its vagueness leading to difficulties with its operationalization.

1.7 Although superdiversity tries to capture the intersection of various overlapping dimensions of difference, in practice superdiversity research often focuses on more subtle and individualised ethno-cultural difference. We argue that despite the opposition to the concept and contestation over its value, 'superdiversity' offers potential as a useful lens through which to capture multi-dimensional perspectives and the way these converge to make up increasingly differential experiences of the social world. The theory and methods through which to advance such knowledge have however yet to be further developed.

1.8 The aim of the paper is to consider different methodological approaches to researching migrants in superdiverse and fluid societies and the scope for methodological hybridity through bringing together different 
methods to develop alternative approaches to research. The paper starts with reflections on the limitations of groupist research designs, which focus on specific ethnic or racial groups who are essentialised as their members are perceived as being predominantly like each other and different from non-members. It calls for multidimensional methodologies that reflect the levels of socio-demographic complexity experienced in contemporary societies. The paper then moves on to discuss a number of specific methodological challenges associated with complex and dynamically changing populations, including sampling and access to different migrants. The subsequent part of the paper discusses the adoption of more innovative research approaches based on the co-production of research data including techniques such as maximum variation sampling, respondent driven sampling, community research and documents of life approaches, all of which have potential for capturing and understanding the heterogeneity, complexity and fluidity concomitant with superdiversity.

The last part of the paper raises concerns regarding the proposed approaches as well as highlighting their possible outcomes, such as the engagement of the individuals researched and their empowerment through the co-production of research data.

\section{Rethinking approaches to social research in superdiverse contexts}

2.1 Traditionally, diversity research originating from a range of academic disciplines, including Sociology, with its roots in the Chicago School of the 1930s, has tended to be dominated by an ethno-focus (Shiller and \&CCEDIL;ağlar 2013; Shiller et al.2006). Previous research was often based on the underlying assumption that individuals from the same ethnic background engage in the same cultural practices and share the same identities, when in reality vast variations can and do exist within different migrant populations. There has been growing criticism of methodological ethnicism which has tended to essentialise populations into ethnic pigeonholes based on the premise of homogeneous cultures and identities. Brubaker (2006) challenges this pervasive and common sense 'groupism' and shows how ethnic diversity is routinely constructed and framed as the struggles of internally homogeneous and externally bounded ethnic groups, which contributes to the reification of ethnic groups conceived as entities and cast as actors. As Meissner and Vertovec (2015) similarly acknowledge, much of the history of migration studies consists of research focused on particular ethnic or national groups and the processes of their integration, separation or assimilation in destination countries. Developing thinking and debates about superdiversity has called for researchers to move beyond an ethnically focused approach to migrants and so-called host societies alike, towards multi-dimensional methodologies which acknowledge the myriad of different factors that may come together to influence the lived experience of individuals from different backgrounds, and appreciate the levels of heterogeneity, complexity and fluidity that have become the reality (Vertovec and Wessendorf 2010).

The concept of 'superdiversity' is related to earlier debates on transnationalism. At the end of the twentieth century it was recognised that the two predominant models of migration, namely settlement immigration and temporary migration, were insufficient for the analysis of contemporary migration (Castles 2002). Past research has highlighted that migrants often maintain relations with two or more countries and live in transnational social spaces. This type of migratory pattern has been described as a system of social relations that cross geographical, administrative, cultural and social borders (Glick Schiller 2003). These emergent migratory patterns have resulted in the development of a new transnational perspective (Faist 2000;Levitt and Glick Schiller 2004). At the same time nation states have begun to be undermined by both external and internal forces; top-down and bottom-up processes related to diversity, globalisation and transnationalism which have led to questioning the 'nation-state-society' paradigm (Favell 2010). A transnational perspective not only recognises the realities of contemporary migration but also helps overcome 'methodological nationalism' and the prior tendency to concentrate on states as a natural unit of analysis, which has limited research within the context of a given destination country (Wimmer and Glick Schiller 2002). A transnational research perspective also encourages the examination of processes which spread across borders by using, for example, multi-site methodology. Moreover, as Meissner and Vertovec point out that '...finding parallels in different contexts will contribute to the understanding why certain saliencies of difference, and their intersections, prevail in particular contexts' (Meissner 2015:11). super-mobility. Much of the developed world has entered an era of 'liquid' societies, which Bauman (2000) associates with ongoing institutional transformations, complex processes and drifting individuals. Following on from this Urry (2000) suggests that ongoing mobility, flows and networks as well as overlapping forms of stratification make classic concepts of society and nation problematic. As a result he proposes the development of an alternative approach to the study of society. Urry (2000) refers to this alternative approach as the sociology of mobility, which he claims as a new conceptual approach built around the categories of networks, mobility and fluidity. In a similar vein, Castells (2000) uses the notion of network to capture complex and dynamically changing contemporary society and its endless transformations. 
In order to take forward discussions about increasing population complexity and what this means in relation to the changing conditions of the social world it is important to think about new and different ways that move beyond ethnicity to permit more adequate levels of analysis of the complexities that underpin superdiversity. Similarly, Sigona (2013) argues the need to think about different ways of looking at a society becoming increasingly 'complex, composite, layered and unequal'. Such new levels of population complexity point to the need for multi-faceted research approaches that acknowledge key variables and determinants of superdiversity.

Those interested in applying a 'superdiversity lens' need to consider how a range of possible variables, not just ethnicity and migration backgrounds, converge in different contexts or in relation to different problems or phenomena. Grillo (2015) proposes considering super-diversity's multidimensionality as occurring along many different axes (see Figure 1). The first, most emphasised, axis (x) refers simply to ethnicity; the second axis (y) refers to socio-legal and political status; a third axis (z), distinct from ethnicity, relates to socio-cultural diversity (for example, language and religion), and a fourth axis (w), arguably the most important, refers to diversity of economic status and opportunities for earning a livelihood, that is to say various forms of inequality. It is worth noting that whilst the multidimensional characteristics of superdiversity are now well acknowledged the combinatorial effects warrant further consideration.

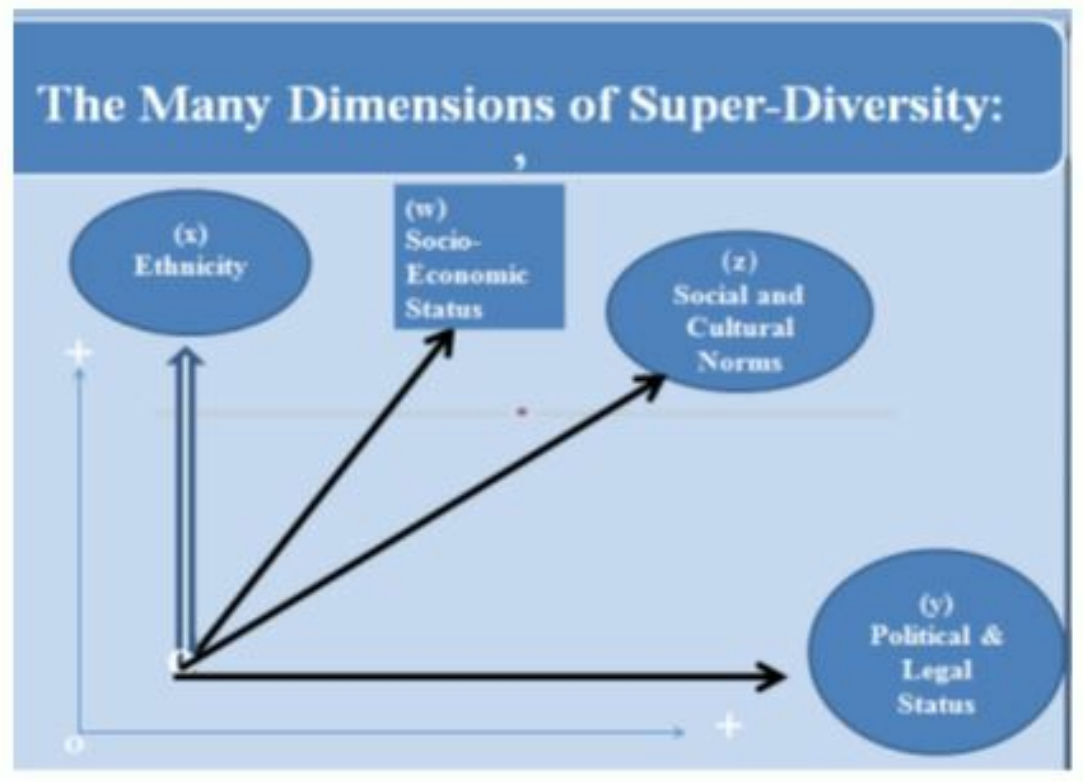

Figure 1. Different dimensions of superdiversity

Source: Grillo (2015)

2.6 Factors such as length of stay and other forms of inequality connected to age, gender, sexuality,

(dis)ability, education, language for example are other important variables that may be more salient markers of lived experience than ethnicity alone. Reflecting on the work of Vertovec (2007) and others (Baycan-Levent 2010; Blommaert 2013b; De Bock 2014), Pride (2015) proposes a multi-dimensional framework to illustrate the interplay between different domains of superdiversity (Figure 2). Each domain is made up of a number of different variables that emerged from the creation of a superdiversity variable index which in total includes over 30 variables. The overlaps in the Venn diagram represent how any combination of domains and variables can come together with no particular hierarchy. Pride (2015) acknowledges that the framework is a starting point from which to develop a more nuanced understanding of the way different domains and variables intersect to influence migrants' lived experiences.

Pride (2015) clusters a number of variables within each superdiversity domain. The individual domain includes personal characteristics such as date of birth, gender, country of origin, ethnicity and religious tradition. The migration domain includes variables such as migration channel, immigration status, date of arrival, intention of length of stay. Pride's (2015) discussion of the migration domain acknowledges the complexity found within migration channels and immigration statuses that give rise to the diversification of legal entitlement and conditions. The socio-economic domain comprises of variables such as education, occupation and income which influence an individual's social and economic position. Pride (2015) goes on to explain how the socio-economic status of a community falls into the space/ place domain to refer to attributes that reflect a community as a whole. These include, ethnic makeup of a community, diversity within groups within a community, rates of population turnover, deprivation rates, places of worship or other physical space available to community members. The 
space/ place domain offers additional variables that can have significant yet variable impact on individuals when they intersect with other superdiversity domains. Finally, the household domain refers to the characteristics and relationships within a household such as number of residents, dependent members, languages spoken. Pride's (2015) multidimensional framework offers potential for researchers interested in better understanding the notion of superdiversity and those engaged in different aspects of policy research in superdiverse contexts.

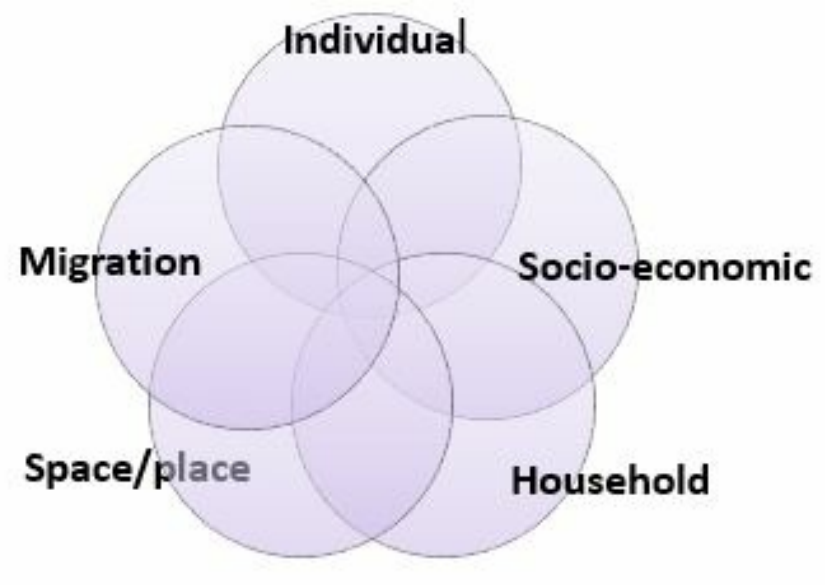

Figure 2. Superdiversity dimensions

Source: Pride (2015)

An emphasis on multi-dimensionality shifts the lens of analysis away from ethnicity and migration in order to capture different perspectives and experiences in a given context in order to more accurately represent and understand the 'diversification of diversity' (Vertovec 2012). A broader conceptualisation of superdiversity such as that proposed by Pride (2015) also prompts research that goes beyond traditional intersectionality theory.

Similarities between superdiversity and intersectionality, as well as a substantial potential which could emerge from the combination of these two perspectives, makes it worth highlighting some premises and problems of the latter. Traditional feminist intersectionality theory, historically preceding superdiversity research, tends to focus on the interplay between gender, class and race, in order to examine a range of multivariate relationships. Davis (2008) argues that intersectionality's alleged weaknesses, more specifically its ambiguity and open-endedness, are the secrets to its popularity. Intersectionality as such concentrates on the relationship amongst multiple dimensions and modalities of social relations as itself a central category of analysis. McCall (2005) distinguishes three useful approaches to multiple, intersecting and complex social relations. The first, 'anticategorical complexity', is based on a methodology that deconstructs analytical categories as social life is too complex and fluid. The second, 'intercategorical complexity', adopts existing analytical categories to document relationships of inequality amongst groups and changing configurations of power. The third, 'intracategorical complexity', represents an approach lying between the first two and neither rejects categories nor uses them strategically, examining the boundary-making and boundary-defining process itself and focusing on complexity within groups. McCall (2005) argues the need to develop interesectionality as a methodology defined as a coherent set of ideas about the philosophy, methods, and data that underlie the research process and the production of knowledge. She notes that little discussion of how to study intersectionality, in terms of its methodology, has taken place to date. Thus the scope of intersectionality theory to inform methodological developments has largely been restricted. This restriction is due to the complexity that emerges when the subject of analysis includes multiple dimensions of social life and categories of analysis. This complexity has led to favouring methodologies that more naturally lend themselves to the study of complexity and rejecting those that may be perceived as too reductionist and simplistic or too difficult. Similar types of development and limitations can be seen in superdiversity research. Developing a methodology of superdiversity which could embrace aspects of difference is therefore an equally needed endeavour.

2.10 A counter criticism can be levelled at wider conceptualisations of superdiversity regarding their ability to allow for 'conscientious empirical application' (Meissner and Vertovec 2014: 4). However, whilst knowledge of which variables are most significant in a given context and across disciplines is at various stages of development, we argue that research approaches that help to operationalise superdiversity will undoubtedly help to identify the parallels that Meissner and Vertovec (2014) consider important in strengthening our understanding of difference in particular contexts. We further argue that wherever possible lessons should be taken from past research in order to develop strategies and checks to avoid essentialising salient categories either temporally or spatially. Working in multidisciplinary teams that bring a range of perspectives to the study of superdiversity we also argue offers a productive way to develop theoretical knowledge and advance research designs. 
The multidimensionality of diversity leads to problems not only with researching superdiversity but also representing it. However, new advanced multidimensional methods of visualising superdiversity are developing (Hiebert 2015). An example is the Globaldivercities project where visual anthropology (photography, film, video) is used alongside geographic visualisation A key technique relates to geographical visualisation including innovative mapping, which uses socio-economic and demographic data visualised in graphic form and integrated into GIS maps along with visual anthropology material (Vertovec 2011). Linguistic landscaping offers a further technique that entails compiling photographs and other evidence of different languages with geo-referenced notes on linguistic observation (cf. Blommaert 2013a). The development of overlay methods for combining material related to mental maps, behavioural maps, physical maps, visual images, data visualisation and ethnographic observations offers potential for each source and type of data to inform the analysis of another and together create a unique picture and multidimensional analysis (Hiebert 2015).

2.12 In summary, similar in some ways to the research that emerged from the Chicago School in the 1920s 1940's (cf. Thomas 1921; Wirth 1928; Thomas and Znaniecki 1927; Whyte 1943), many studies undertaken in superdiverse contexts involve smaller, less visible and more fragmented communities than previous research carried out in the post-colonial period which focused on larger more established ethnic groups. This raises important questions regarding the appropriateness of different research methods as well as the associated sampling and access techniques. In particular the ability of different methodologies to deal with frequently encountered practical difficulties in the field relating to trust and rapport, language barriers, translation and interpretation issues. Moving beyond the commonly used ethnographic approaches, based on Geertz's (1973) 'thick description', the following section will present four different revived approaches which offer promising tools for researching migrants in the context of superdiversity. The research approaches outlined include, maximum variation sampling, respondent driven sampling, community research and documents of life method. They can be seen as approaches which increasingly appreciate diversity and engage research populations, in turn empowering them by offering them the opportunity to become co-producers of knowledge.

\section{Alternative approaches to studying superdiverse societies}

3.1 Maximum variation sampling (MVS) is a type of focused sampling in which researchers aim to study very different individuals, in order to capture common patterns that emerge from great variation as representing core or central experiences (Patton 1999). MVS allows description and understanding of variation within a group. Instead of assuring representativeness through equal probabilities, MVS tries to obtain it by including a wide range of extremes. Although it may be questioned to what extent the 'aggregate' answers of individuals at the extremes can be in line with those of the whole population. The research design gives an opportunity both to perform in-depth analysis within the chosen populations and to minimise the effect of specific ethnic and cultural factors by means of maximum variation. The use of MVS is useful in addressing the essentialist critique, as well as providing the opportunity to include difficult to classify, 'border' cases that exist in a contemporary complex and diverse society. This MVS method is therefore highly appropriate for examining the heterogeneity of migrants as the approach goes beyond sampling based on ethnic groups to include target populations that are as diverse as possible. An example of such research is the study by Phillimore et al. (2014) into the relationship between migrants' social networks and their access to integration resources. Phillimore et al. (2004) examined the experiences of 37 migrants in four different cities in the UK. The sample comprised of individuals with different countries of origin, ethnicities, migration and socio-economic statuses, life stages and faiths, and manifesting various levels of skills in the English language. The idea behind this methodological choice was that if people are as different as possible any commonalities identified would provide potential for generalisation, resulting in a solid foundation for the development of policy recommendations.

In addition to MVS, respondent driven sampling (RDS) has become recognised over the past decade as a beneficial option for rigorous sampling of hard-to-reach and 'hidden' populations (Johnston and Sabin 2010). Examples of such populations are: members of the LGBT community, intravenous drug users and populations at high risk of HIVIAIDS (Heckathorn et al. 2002, Ramirez-Valles et al. 2005). This innovative approach has also been successfully applied to migration research (Tyldum and Johnston 2014). In general, RDS is a chain-referral technique for estimating traits in difficult-to-reach populations in which participants recruit their peers. The research starts with the selection of 'seeds' which ensures that different types of individuals' characteristics of the population studied are represented in the research sample. A dual system of structured incentives employed in this technique overcomes the deficiencies of other types of sampling (such as snowball and other chain-referral samples, the key-informant approach, and targeted sampling) which reflect the sampling choices made by the researcher and are influenced by the visibility and accessibility of respondents. With the RDS technique the composition of the respondent driven sample is independent of the researcher and those initial seeds (Heckathorn 1997). Ensuring the recording of a detailed track of recruitment (who recruited whom and what were their number of social contacts), a mathematical model based on a synthesis and extension of Markov's chain theory and biased network theory can be employed to weight the sample to compensate for non-random 
recruitment patterns and thus provide both unbiased population estimates and measures of the precision of those estimates (Salganik and Heckathorn 2004). Despite some concerns around accuracy and representativeness, and the costs of this method, RDS has gained popularity in social research because it enables advanced quantitative analysis to be employed. In addition, the involvement of participants in recruiting other respondents affords them some power in the research process as they cease to be only passive participants in the research. Furthermore recruitment that stems from a known individual can help to overcome some of the difficulties associated with trust in the research process and additionally motivate subsequent participants to engage in the research. RDS has been shown to have promise in surveys of Polish migrants in Dublin, Oslo and Reykjavik (Tyldum and Johnston 2014). A modification of RDS has also been successfully employed in the study of Polish and Pakistani migrants in London (Platt et al.2015). In this study the anonymity and respondent-controlled recruitment used in traditional RDS was replaced with a researcher-led referral method entailing the direct collection of contact details of referrals both through call-backs and within the survey questionnaire.

Community and practitioner research methodologies represent an alternative approach which is particularly relevant to research into migrants in the context of superdiversity. Vertovec (2007) calls for greater recognition of the need to find new ways to give voice to diverse minority groups. Community research not only offers opportunities for community members to recruit participants, as with RDS, but for them to become involved in all stages of the research process. We define community research as 'the practice of engaging community members as co-researchers to research issues within their own communities with a view to accessing community specific knowledge' (Goodson and Phillimore 2012: 4). We understand community research not only as a type of research but as a methodological approach that is particularly valuable for studying superdiversity, which can also benefit the individuals and communities involved. There are several examples of community research projects involving migrant communities. For example Atfield et al. 's (2012) work with refugees (2012) and Brown et al.'s (2012) study with Gypsies and Travellers in the UK.

Community research has been proven to have the potential to both empower and build the capacity of communities under study (Goodson and Phillimore 2012). In this respect community research can be located under the participatory action research umbrella (PAR) which can take a range of forms and involve community members to varying degrees in the research process as Figure 3 illustrates.

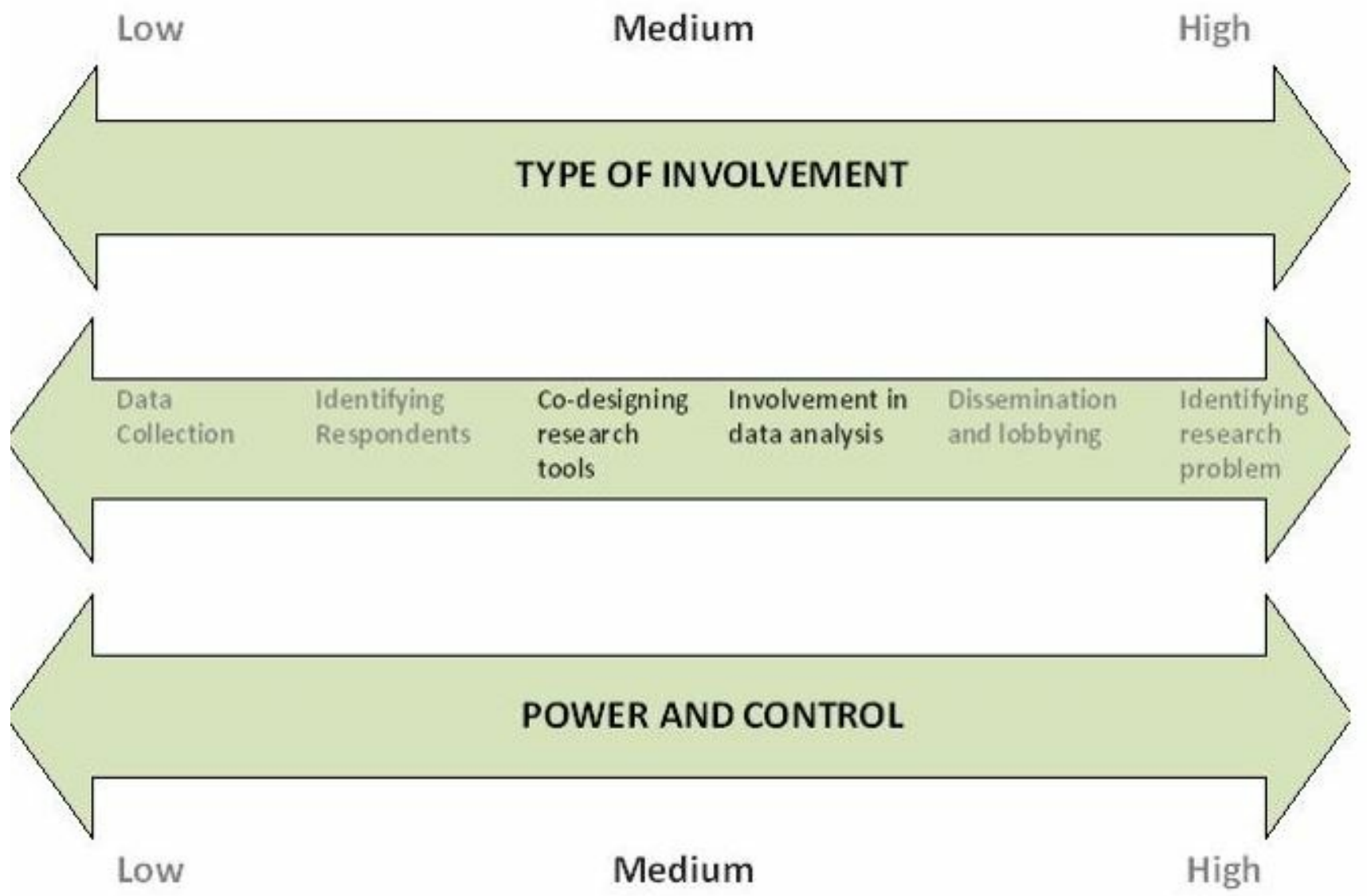


Figure 3. The relationship between community researchers' level of involvement and power and control Source: Goodson and Phillimore (2012: 6)

At its best community research is concerned with participation, partnerships and action through research in communities. The aim of such research is not only to understand the world but also to help understand how best to make changes collaboratively that meet the needs of those involved. We should however be mindful in our research designs about the way we define 'community'. In order to avoid being led into further 'ethnic' traps we need to think strategically about how to operationalise superdiversity through the recruitment of community researchers and the sampling of research participants.

The community research approach relies on partnerships between social researchers, grant providers and the group or organisation being engaged. Community research is a way of enabling communities to play an active role in shaping and conducting research. The most constructive use of the approach both from an academic and community perspective is when community researchers are treated as equal partners involved in all stages of the research process from research conception, design, data collection, analysis, interpretation and dissemination. The use of community based knowledge can bring new perspectives to research which is extremely valuable in the creation of new knowledge that provides deeper and richer insights for theory building or to stimulate action orientated outcomes and policy change. Working with community researchers can help overcome problems associated with accessing 'hidden' communities. The approach has been described as offering a 'doorway' into the lives of those who would otherwise remain undiscovered by traditional research teams. A range of reasons have been identified which to some extent explain why the experiences of certain migrant groups remain undiscovered, these include: community members' unwillingness to speak to 'officials' due to a lack of trust regarding the way research data may be used; language barriers, lack of confidence, lack of rapport and inability of researchers to probe sensitive or personal issues (cf. Goodson and Phillimore 2010). Whilst community research is not without its challenges it offers a promising approach to undertaking research in superdiverse contexts as community researchers' expert knowledge of their community helps to enhance the validity of research findings. Community researchers' language skills, social networks and social capital also provide invaluable assets to research teams interested in exploring the lives of individuals across a range of diverse communities.

The documents of life approach offers further potential for researching migrants in a superdiverse context. As with a community research methodology, the documents of life approach aims to empower research participants by granting them the space to express their own stories in their own way. Doing life story research has been postulated by Plummer (2001) as a response to the emergence of the reflective society and 'the narrative turn' in social science. Furthermore, Plummer (2001) sees the documents of life as a response to, and critique of, anti-humanist methodologies. By doing life story research, Plummer (2001) tries to promote critical humanism which pays tribute to human subjectivity and creativity, and deals with human experience including the self-awareness of researchers. The documents of life approach, which originates from narrative and biographical inquiry, typically uses life stories and other personal documents such as 'natural', researched and reflective stories, autobiographies, diaries and photos (Stanley 2013). In addition emails, Internet fora and Web blogs can be used as data for analysis. There is great scope to combine the documents of life approach with a community research methodology to encourage participants to be both actively and creatively engaged in research. approach which can be traced back to classical biographical research into migrants' experiences in the early $20^{\text {th }}$ century. For example, Thomas and Znaniecki's (1927) seminal work The Polish Peasant in Europe and America skilfully adopts the approach to offer rich accounts and insights of Polish migrants and their families in America based on a range of personal documents. Oral history may also be viewed as a type of documents of life approach. As Herbert (2008) points out, oral history allows the production of a specific kind of knowledge, which is:

\footnotetext{
'... attentive to the diversity of experiences, that focuses attention on social networks, family and household dynamics and privileges the agency of the respondents. Rather than being positioned as the object of the research, the respondent is seen as actively engaged in the process of constructing their own narrative, within which they bestow particular events and characters with meanings as they attempt to evaluate and make sense of their past. The focus is on the essence of human creativity and subjectivity, including motivations, perceptions and feelings; in short, on the
} 
For example, Herbert (2008) uses in-depth life-story interviews and oral history archives to offer a historically grounded analysis of the human experiences of migration and explores the impact of South Asian migration from the 1950s onwards on both the white, British-born population and the migrants themselves.

3.10 Oral history and other documents of life stories can be seen as particularly valuable for research with migrants, whose experiences are unique and difficult to access via conventional research and documentary sources, as the study by Benmayor and Skotnes (1994) for example illustrate. Whereas in community research the community researchers are trained and guided to do research led by scholars, the documents of life approach focuses more on 'natural' testimonies or 'stimulated' documents (for example by organising and presenting photos/diaries/letters). The documents of life approach allows unheard voices to be heard, highlights unclassified cases and uncovers diversity and complexity. This is an approach which empowers groups and individuals to recognise their experience and engages them in the co-production of knowledge in a more organic way, with participants having increased levels of power and control over the type and format of data collected.

\section{Discussion}

4.1 This paper discusses the challenges, methods, concerns and promises related to conducting research in superdiverse contexts and uses superdiversity as a new lens through which we can examine complex and multilayered reality. The text highlights the growing complexity of contemporary urban populations, and other factors such as ethnicity, length of settlement, legal status, identities, attitudes to acculturation, religious affiliations, socio-economic situation, lifestyles and lived experience. This paper also sets out how diversity and mobility of people pose new challenges for social research. It further reflects on the limitations of ethnicism in informing research design and calls for multidimensional methodologies alongside more participatory research approaches. The article proposes the adoption of a range of research approaches that offer potential in terms of better capturing and understanding the heterogeneity, complexity and fluidity concomitant with superdiversity, as well as engaging community groups and other stakeholders in research.

4.2 Although this paper presents different understandings of superdiversity (for example as a descriptive term, a sensitising concept, a theoretical framework) it mainly problematises superdiversity in relation to methodology. The article presents four different revived or innovatively used approaches which offer promising tools for studying migrants in the context of superdiversity: maximum variation sampling, respondent driven sampling, community research and the documents of life method.

The paper highlights the potential that MVS has for sampling superdiverse communities and discusses the challenges of engaging hard to reach communities. The importance of methodological approaches that appreciate the significance of trust and rapport building in the research process in order to secure the engagement of community members from diverse backgrounds is also stressed. Co-production methodologies can be extremely valuable to facilitate the 'buy in' and involvement of community members in the production of knowledge. The scope that RDS has for the production of quantitative data and the potential that working with teams of community researchers has for generating in-depth qualitative insights on topics that would remain inaccessible to most academic and professional researchers has been highlighted. Active engagement in community research as well as documents of life and oral history approaches have particular merits in the context of superdiversity, as they offer potential for creative expression and giving a real voice to potential research participants.

4.4 Future research concerning the process, conditions and implications of superdiversity may benefit from considering the scope of combining some of the techniques outlined in this paper to develop alternative research approaches. For example, combining the RDS approach with the principles of MVS to prompt the selection of the most diverse seeds possible and/or combining documents of life and oral history methodology with a community research approach to illuminate new insights impossible to uncover using more conventional research methods. The combination of all four approaches would provide opportunities to undertake complex, mixed method studies. Furthermore, working with teams of researchers across multiple sites in different countries would enable the inclusion of a transnational perspective and thus challenge the limitations of the nation-state-society paradigm (Favell 2010). This approach, although in the early stages of development, is also being adopted as part of a large European research programme examining welfare bricolage in superdiverse contexts in four European countries: the United Kingdom, Sweden, Germany and Portugal (Phillimore et al.2015). The insights gained from this project will further advance methodological thinking as well as help to establish the epistemological and ontological standpoint of this emerging paradigm. 
immense scope to advance superdiversity research. The approaches discussed in this paper all offer more voice and representation to those who are often overlooked or marginalised in research. Furthermore, the adoption of co-production techniques offers potential to help dilute prevailing clichés and stereotypes about migrants, which, against the backdrop of the migrant crisis and growing anti-migrant sentiments, is critically needed. There is scope with all the approaches presented in this paper to actively engage migrant communities and practitioners in the process of research and production of knowledge, thus providing more power to those concerned to shape research agendas. The approaches presented here, particularly RDS, can also be adapted to fit with a transnational perspective and move research beyond methodological nationalism. For example, in RDS migrants may be permitted to recruit their peers beyond their current country of residence.

Researchers working in superdiverse contexts should however remember that capturing heterogeneity and focusing on specificities should not be done for its own sake but consideration should be given to theoretical, practical and political developments that can be made in order to advance thinking in this area. The significance of multi-dimensionality in relation to intersectionality and inequality, for example, is an important issue that warrants further attention by researchers interested in adopting a superdiverse lens. Finally, another important methodological issue that has not been addressed in this paper due to limited space is the problem of generalisability and relativism, which need careful consideration by researchers working in superdiverse contexts.

\section{Notes}

$1 \quad$ The A8 countries are a group of eight countries that joined the European Union during its 2004 enlargement. The A8 group comprises the Czech Republic, Estonia, Hungary, Latvia, Lithuania, Poland, Slovakia and Slovenia. The A2 countries, Bulgaria and Romania, joined the European Union in 2007.

\section{References}

ARNAUT, K. and Spotti, M. (2014) Superdiversity discourse, Working Papers in Urban Language and Literacies No 122, King's College London http:

//www.kcl.ac.uk/sspp/departments/education/research/ldc/publications/workingpapers/thepapers/WP122.pdf.

ATFIELD, G., Brahmbhatt, L., Hakin, H. and O'Toole, T. (2012) Involving community researchers in refugee research in the UK. In Goodson, L. and Phillimore, j. (ed) Community research for participation: from theory to method. Bristol: Policy Press.

BACK, L. (2015) Losing culture or finding superdiversity, Discover Society, 20. http://discoversociety.org/2015/05/05/losing-culture-or-finding-superdiversity-2/.

BAUMAN, Z. (2000) Liquid Modernity. Cambridge, UK: Polity.

BAYCAN-LEVENT, T. (2010) Diversity and Creativity as Seedbeds for Urban and Regional DynamicsEuropean Planning Studies 18 (4): p. 565-594. DOI: 10.1080/09654311003593663.

BENMAYOR, R. and Skotnes, A. (1994) Migration and identity. Oxford: Oxford University Press.

BLOMMAERT, J. (2013a) Ethnography, superdiversity and linguistic landscapes: Chronicles of complexity. Bristol: Multilingual Matters.

BLOMMAERT, J. (2013B) The second life of old issues: How superdiversity 'renews' things.Cultural Studies No 59, Tilburg University https: //www.tilburguniversity.edu/upload/74c04845-e6f0-4899-977ede5bf1b8f9da_TPCS_59_Blommaert.pdf.

BROWN, P., Scullion, L. and Niner, P. (2012) Community research with Gypsies and Travellers inthe UK: highlighting and negotiating compromises to reliability and reliability. In Goodson, L. and Phillimore, J. (ed) Community research for participation: from theory to method.Bristol: Policy Press.

BRUBAKER, R. (2006) Ethnicity without groups. Cambridge, MA: Harvard University Press. 
CASTELLS, M. (2000) The Rise of the network society: the information age: economy, society and culture. Oxford: Blackwell.

CASTLES, S. (2002) Migration and community formation under conditions of globalization/nternational Migration Review 36(4): p. 1143-1168. [doi:10.1111/j.1747-7379.2002.tb00121.x]

COHEN, P. (1994) Home Rules: Some Reflections on Racism and Nationalism in Everyday Life,Dagenham: New Ethnicities Unit University of East London.

DAVIS, K. (2008) Intersectionality as buzzword, Feminist Theory 9(1): p. 67-85. [doi:10.1177/1464700108086364]

DE BOCK, J. (2014) Not all the same after all? Superdiversity as a lens for the study of past migrations.Ethnic and Racial Studies, DOI: 10.1080/01419870.2015.980290 [doi:10.1080/01419870.2015.980290]

ENGBERSEN, G., Leerkes, A., Grabowska-Lusi?ska, I, Snel E. and Burgers, J. (2013) A typology of labour migration. On the differential attachments of migrants from Central and Eastern Europe. Journal of Ethnic and Migration Studies, 39(6): p. 959-981.

FAIST, T. (2000) The volume and dynamics of international migration and transnational social spaces. Oxford: University Press. [doi:10.1093/acprof:oso/9780198293910.001.0001]

FAVELL, A. (2010) Integration and nations. In J. Rath and M. Martiniello (eds)Selected Studies in International Migration and Immigrant Incorporation. Amsterdam: Amsterdam University Press.

GEERTZ, C. (1973) Thick description: toward an interpretive theory of culture. In C. GeertzThe interpretation of cultures: selected essays. New York: Basic Books.

GLICK SCHILLER, N. (2003). The centrality of Ethnography in the study of transnational migration. In N. Foner (ed.) American Arrivals, Santa Fe. The New Immigration School of American Research Press.

GOODSON, L. and Phillimore, J. (2010) A community research methodology: working with new migrants to develop a policy related evidence base. Social Policy and Society9 (4).

[doi:10.1017/S1474746410000217]

GOODSON, L. and Phillimore, J. (2012) Community research for community participation: from theory to method. Bristol: Policy Press.

GRILLO, R. (2015) Reflections on super-diversity by an urban anthropologist, or 'superdiversity so what?'. Superdiversity Academy, Berlin, 8-10 April 2015 (unpublished conference paper) https: //www.academia.edu/12392425/_Reflections_on_SuperDiversity_by_an_Urban_Anthropologist_or_Superdiversity_So_What.

HECKATHORN, D. (1997) Respondent-driven sampling: a new approach to the study of hidden populations. Social Problems, 44(2): p. 174-199.

HECKATHORN, D., Semaan, S., Broadhead, R. and Hughes, J. (2002) Extensions of respondent-driven sampling: a new approach to the study of injection drug users aged 18-25. AIDS and Behavior, 6 (1): $p$. 55-67. [doi:10.1023/A:1014528612685]

HERBERT, J. (2008) Negotiating boundaries in the city: migration, ethnicity, and gender in BritainAldershot: Ashgate.

HIEBERT, D. (2015) Ethnocultural Minority Enclaves in Montreal, Toronto and Vancouver. IRPP Study, no. 52, August 2015

HUMPHRIS, R. (2015) Race and superdiversity - what are the links? IRiS Key Concept roundtable series report, http://www.birmingham.ac.uk/schools/education/research/crre/news/2015/race-and-superdiversity-irisroundtable-series.aspx.

KNOWLES, C. (2010) Theorising Race and Ethnicity: Contemporary Paradigms and Perspectives. In: P. H. Collins and J. Solomos The SAGE Handbook of Race and Ethnic Studies, SAGE [doi:10.4135/9781446200902.n3]

JOHNSTON, L. and Sabin, K. (2010) Sampling hard-to-reach populations with respondent driven sampling. Methodological Innovations Online, 5(2): p. 38-48. 
LEVITT, P. and Glick Schiller, N. (2004) Conceptualizing simultaneity: a transnational social field perspective on society. International Migration Review, 38(3): p. 1002 -1039. [doi:10.1111/j.1747-7379.2004.tb00227.x]

MCCALL, L. (2005) The Complexity of Intersectionality, Signs: Journal of Women in Culture and Society30(3), p. 1771-1800.

MEISSNER, F. and Vertovec, S. (2015) Comparing super-diversity. Ethnic and Racial Studies, 38(4): p. 541-555. [doi:10.1080/01419870.2015.980295]

ONS (2013) Local characteristics on migration for output areas in England and Wales Office for National Statistics. Released 26 September 2013. London: Office for National Statistics.

PATTON, M. (1999) Qualitative evaluation and research methods, Beverly Hills, CA: Sage.

PHILLIMORE, J. (2015) Delivering maternity services in an era of superdiversity: the challenges of novelty and newness. Journal of Ethnic and Racial Studieshttp: //www.tandfonline.com/doi/full/10.1080/01419870.2015.980288.

PHILLIMORE, J., Humphris, R. and Khan, K. (2014) Migration, networks and resources: the relationship between migrants' social networks and their access to integration resources. http: //king.ismu.org/wpcontent/uploads/PhillimoreHumphrisKhan_InDepthStudy.pdf.

PHILLIMORE, J., Bradby, H., K, M., Padilla, B., Brand, T., Cheung, S., Pemberton, S. and ZeebH. (2015) Understanding healthcare practices in superdiverse neighbourhoods and developing the concept of welfare bricolage: Protocol of a cross-national mixed-methods study. BMC International Health and Human Rights 15, DOI: 10.1186/s12914-015-0055-x.

PLATT, L., LUTHRA, R. and FRERE-SMITH, T. (2015) Adapting chain referral methods to sample new migrants: possibilities and limitations, Demographic Research, DOI; 10.4054/DemRes.2015.33.24 [doi:10.4054/demres.2015.33.24]

PLUMMER, K. (2001) Documents of life-2: an invitation to a Critical Humanism Sage: London.

PRIDE, M. (2015) Measuring superdiversity: constructing a theoretical multi-dimensional framework. Unpublished MA dissertation. School of Social Policy, University of Birmingham.

RAMIREZ-VALLES, J., Heckathorn, D., Vázquez, R., Diaz, R. and Campbell, R. (2005) From networks to populations: the development and application of respondent-driven sampling among IDUs and Latino gay men. AIDS and Behavior, 9: p. 387-402.

SALGANIK, M. and Heckathorn, D. (2004) Sampling and estimation in hidden populations using respondentdriven sampling. Sociological Methodology, 34: p. 193-239. [doi:10.1111/j.0081-1750.2004.00152.x]

SASSEN, S. (1996) Analytic borderlands: race, gender and representation in the new city. In A. King (ed.) Representing the city: ethnicity, capital and culture in the twenty-first century metropolis.London: Macmillan.

SCHILLER, N. and Çağlar, A. (2013) Locating migrant pathways of economic emplacement: Thinking beyond the ethnic lens. Ethnicities, 13(4): p. 494-514. [doi:10.1177/1468796813483733]

SCHILLER, N., Çağlar, A., and Guldbrandsen, T. (2006) Beyond the ethnic lens: Locality, globality, and bornagain incorporation. American Ethnologist, 33(4): p. 612-633.

SEPULVEDA, L., Syrett, S., and Lyon, F. (2011) Population superdiversity and new migrants' enterprise: the case of London. Entrepreneurship and Regional Development23(7-8): p. 469-497. [doi:10.1080/08985620903420211]

SIGONA, N. (2013) Imagining a new research agenda in an era of superdiversity, in postcards from ... weblog, 25 October. http: //nandosigona.wordpress.com/2013/10, date accessed 19 June 2015.

SMITH, A. (2014). Rethinking the 'everyday' in 'ethnicity and everyday life',Racial and Ethnic Studies, [doi:10.1080/01419870.2014.987307]

STANLEY, L. (2013) Documents of life revisited. Aldershot: Ashgate.

THOMAS, W. (1921) The Immigrant Community. Chicago: University of Chicago Press.

THOMAS, W. and Znaniecki, F. (1927) The Polish Peasant in Europe and America. New York: Alfred A. Knopf. 
THOMAS, A. (1999) Moving stories: oral history and migration studies.Oral History 27 (1), p. 24-37.

TYLDUM, G. and Johnston, L. (2014) Applying respondent driven sampling to migrant populations: lessons from the field. London: Palgrave Macmillan.

URRY, J. (2000) Sociology beyond Societies. London: Routledge.

VERTOVEC, S (2006) The emergence of super-diversity in Britain. ESRC Centre on Migration, Policy and Society Working Paper WP-06-25.

VERTOVEC, S. (2007) Super-diversity and its Implications. Ethnic and Racial Studies30(6): p. 1024-1054. [doi:10.1080/01419870701599465]

VERTOVEC, S. (2010) Super-diversity and its implications. In S. Vertovec (ed.)Anthropology of migration and multiculturalism: New directions, London: Routledge. p. 65-96.

VERTOVEC, S. (2011) Migration and new diversities in global cities: comparing, conceiving, observing and visualising diversification in urban public spaces. MMG Working Paper.

VERTOVEC, S. (2012) Diversification and 'diversity': configurations, representations and encounters. Guest lecture, Institute for Research into Superdiversity (IRiS) at the University of Birmingham, 24th October 2012.

VERTOVEC, S. and Wessendorf, S. (2010) The multiculturalism backlash. European discourses, Policies and practices. London: Routledge.

WESSENDORF, S. (2010) Commonplace diversity: Social interactions in a super-diverse context.Working Paper of the Max Planck Institute for the Study of Religious and Ethnic Diversity (WP 10-11).

WHYTE, W. (1943) Street Corner Society: The Social Structure of an Italian Slum. Chicago: University of Chicago Press.

WIMMER, A. and Glick Schiller, N. (2002) Methodological nationalism and beyond: nation-state building, migration and the social science. Global Networks, 2(4): p. 301-334.

WIRTH, L. (1928) The Ghetto. Chicago: University of Chicago Press. 